\title{
Survival Advantage of Non-hispanic White Patients with Pancreatic Head Carcinoma: A Population-based Study
}

\author{
Hongyu Yu' ${ }^{1}$, Chengzhuo $\mathrm{Li}^{2,3}$, Qihui $\mathrm{Wu}^{1}$, Jukun $\mathrm{Su}^{1}$, Ankang Liu ${ }^{1}$, Qiqi Ke ${ }^{1}$, Qiaohong Yang ${ }^{1}$, \\ ${ }^{1}$ School of Nursing, Jinan University, Guangzhou, China \\ ${ }^{2}$ School of Public Health, Xi'an Jiaotong University Health Science Center, Xi'an, China \\ ${ }^{3}$ Department of Clinical Research, The First Affiliated Hospital of Jinan University, Guangzhou, China
}

Email address:

yqiaohong@163.com (Qiaohong Yang)

${ }^{*}$ Corresponding author

\section{To cite this article:}

Hongyu Yu, Chengzhuo Li, Qihui Wu, Jukun Su, Ankang Liu, Qiqi Ke, Qiaohong Yang. Survival Advantage of Non-hispanic White Patients with Pancreatic Head Carcinoma: A Population-based Study. American Journal of Biomedical and Life Sciences.

Vol. 9, No. 1, 2021, pp. 58-68. doi: 10.11648/j.ajbls.20210901.18

Received: January 10, 2021; Accepted: January 20, 2021; Published: February 2, 2021

\begin{abstract}
Numerous studies have shown that racial health disparities in gastroenterology and hepatology, but little is known about its effect on pancreatic head carcinoma (PHC). The aim of the present study was to determine whether racial disparities in the overall survival (OS) and cancer-specific survival (CSS) rates exist among US patients with PHC. The SEER database was searched for US residents who had been diagnosed with PHC from 2007 to 2015. The outcomes for 9724 Hispanic white (HW) patients and their non-Hispanic white (NHW) counterparts were compared using Kaplan-Meier survival and Cox regression analyses. We found that race affected both OS and CSS. The 5-year OS rate was worse for HW patients $(45.9 \%)$ than for NHW patients ( $49.6 \%, \mathrm{P}<0.001)$, as was the 5 -year CSS rate $(39.8 \%$ versus $44.0 \%, \mathrm{P}=0.002)$. Race appeared to be an independent prognostic factor for PHC in the multivariate analysis, with NHW patients showing superior OS ( $\mathrm{P}=0.007)$ and CSS $(\mathrm{P}=0.037)$ compared with HW patients. Subgroup analysis showed that race influenced survival among patients who received surgery, enjoyed Medicaid, and those at American Joint Committee on Cancer (AJCC) tumor-node-metastasis (TNM) stage II, but not of patients at AJCC TNM stage I, III, or IV and those who did not receive surgery or had no insurance. In short, the survival outcomes for PHC are better for NHW than HW patients. The survival advantage is more skewed towards NHW patients than HW patients with PHC, so culturally appropriate interventions, strengthened preventive services, and additional financial support should focus more on HWs.
\end{abstract}

Keywords: Pancreatic Head Carcinoma, Survival Advantage, SEER, OS, CSS

\section{Introduction}

Pancreatic cancer (PC) is poised to jump from fourth to second place among the factors influencing deaths in the US within the next decade [1]. Most cases of PC are accompanied by metastatic disease, and the efficacy of treatments remain unsatisfactory [2]. Pancreatic head carcinoma (PHC) accounts for $70-80 \%$ of PC patients. The resection rate of $\mathrm{PHC}$ was generally low, with about $60 \%$ of patients not being suitable for surgery due to distant metastasis already being present when the disease is first detected. Moreover, the median survival time of advanced-stage patients is only 6-9 months [3]. No major difference in outcome has been observed between pancreaticoduodenectomy and more-extensive surgery [4]. The 5-year survival rate is just 5-7\% in operative resection cases [5].

Latino Americans constitute the largest minority group in the US population and are expanding rapidly, now accounting for $16.3 \%$ of US residents [6] A pattern of Mexican Americans and other Latino groups having survival advantages over non-Latino whites has been widely reported, which is referred to as the Latino, Hispanic, or epidemiological paradox [7]. The mortality rates are lower among Hispanic whites (HWs) than non-Hispanic whites (NHWs) for conditions such as cancer, heart disease, and chronic lower respiratory disease [8]. Studies are increasingly investigating racial health disparities in gastroenterology and hepatology [9], and race has been 
demonstrated to play a role in survival among these cancer patients [10]. Nevertheless, the relationship between PHC prognosis and race remains to be clarified.

Given the above-mentioned situation, we obtained a large data sample of registered PHC patients in the US from the Surveillance, Epidemiology, and End Results (SEER) database [11]. We divided these patients into certain categories in order to determine whether racial differences exist in overall survival (OS) and cancer-specific survival (CSS) among PHC patients in the US, and particularly whether HWs have a survival advantage.

\section{Material and Methods}

\subsection{Sample Source and Research Design}

The SEER program initiated by National Cancer Institute of the US was used as the source of data in this population-based investigation. The SEER database contains information from 20 registries that account for approximately $28 \%$ of US residents [12]. Subscribers are supplied with detailed patient information regarding demographics, tumor situations, therapies, and outcomes [13].

We enrolled $9724 \mathrm{HWs}$ with $\mathrm{PHC}$ who were diagnosed between 2007 and 2015. These people were sorted in accordance with the primary site labeled as ICD code C25.0 (head of pancreas). Patients were excluded if $\mathrm{PHC}$ was a secondary tumor, the marital and insurance statuses were unclear, or there was no information on pathological grade, surgical management, American Joint Committee on Cancer (AJCC) tumor-node-metastasis (TNM) stage, or vital status. This study focused on determining the OS and CSS defined as the duration from diagnosis to death due to PHC. Death due to PHC was deemed an event. Data were censored when the patient was living or had died of other causes at the last follow-up in 2015.

\subsection{Research Variables}

The following variables were also derived from the SEER database and categorized as indicated within parentheses: diagnosis year (2007-2009, 2010-2012, or 2013-2015), age at diagnosis $(<60,60-74$, or $>74$ years), race (HW or NHW), sex (male or female), tumor grade (I, well differentiated; II, moderately differentiated; III, poorly differentiated; or IV, undifferentiated), marital status (married or unmarried), AJCC TNM stage (I, II, III, or IV), surgery status (yes or no), insurance status (insured, Medicaid, or uninsured), survival time, and vital status.

\subsection{Statistical Analysis}

HWs and NHWs served as fundamental objects of the comparison, and then the patient characteristics in both groups were processed multiple times. First, the continuous variables - age at diagnosis (presented as mean $\pm \mathrm{SD}$ values) and diagnosis duration-conforming to a Gaussian distribution were compared using Pearson's $\chi^{2}$ test, while qualitative data were compared using Fisher's exact test. Second, to calculate OS and CSS values, the Kaplan-Meier method was used along with the log-rank test for distinguishing the Kaplan-Meier curves among subgroups. Cox proportional-hazards models were established to identify significant predictors.

All of the statistical analyses were carried out using SPSS (version 26.0, IBM Corporation), with a probability value below 0.05 considered to indicate a difference that was statistically significant.

\section{Results}

\subsection{Baseline Characteristics}

Table 1 stratifies the baseline characteristics of patients according to race. The patients comprised 1351 (13.89\%) HWs and $8373(86.11 \%)$ NHWs. There were more males than females among both HWs (51.15\% versus $48.85 \%)$ and NHWs $(53.57 \%$ versus $46.43 \%$ ). The HW group comprised considerably fewer unmarried than married patients $(39.90 \%$ versus $60.10 \%)$, as did the NHW group (36.31\% versus $63.69 \%$ ). More NHW than HW patients had received surgery $(63.18 \%$ versus $56.18 \%)$ and were insured $(90.29 \%$ versus $70.17 \%)$. There were significant intergroup differences in age $(P<0.001)$, sex $(P=0.001)$, marital status $(P=0.012)$, AJCC TNM stage $(P<0.001)$, surgery status $(P<0.001)$, and insurance status $(P<0.001)$.

\subsection{Race Effects on $O S$}

Survival varied with race $(P<0.001)$, as indicated by the Kaplan-Meier curve for OS in Figure 1A. The OS duration was longer for NHWs than HWs, with median values of 60 months and 56 months, respectively. Similarly, the 5-year OS rate of NHWs was superior to that of HWs (49.6 versus $45.9 \%)$. Univariate analyses revealed that the significant predictive factors for OS were year of diagnosis $(P<0.001)$, AJCC TNM stage IV $(P=0.002)$, NHW $(P<0.001)$, and uninsured $(P=0.008)$. After subsequent adjustment in the multivariate analysis, all of these variables other than AJCC TNM stage IV $(P=0.372)$ remained significant. Compared with HW patients, NHW patients had positive survival outcomes [hazard ratio $(\mathrm{HR})=0.832,95 \%$ confidence interval $(\mathrm{CI})=0.728-0.951, P=0.007]$ (Table 2).

\subsection{Race Effects on CSS}

The Kaplan-Meier curves for CSS are presented in Figure 1B. The 5-year CSS rate was worse for HW than NHW patients $(44.0 \%$ versus $39.8 \%, P=0.001$ in log-rank test). Univariate analyses revealed that all variables were significant predictive factors for CSS, with the exception of male $(P=0.085)$, tumor grades II, III, and IV $(P=0.915,0.613$, and $P=0.625$ respectively), AJCC TNM stages II and III ( $P=0.228$ and 0.292 respectively), and unmarried $(P=0.153$ ). After subsequent adjustment in the multivariate analysis, all of these variables other than AJCC TNM stage IV $(P=0.747)$ remained significant. The survival outcomes were better for NHWs than their HW counterparts $(\mathrm{HR}=0.879,95 \%$ $\mathrm{CI}=0.779-0.992, P=0.037$; Table 3). 

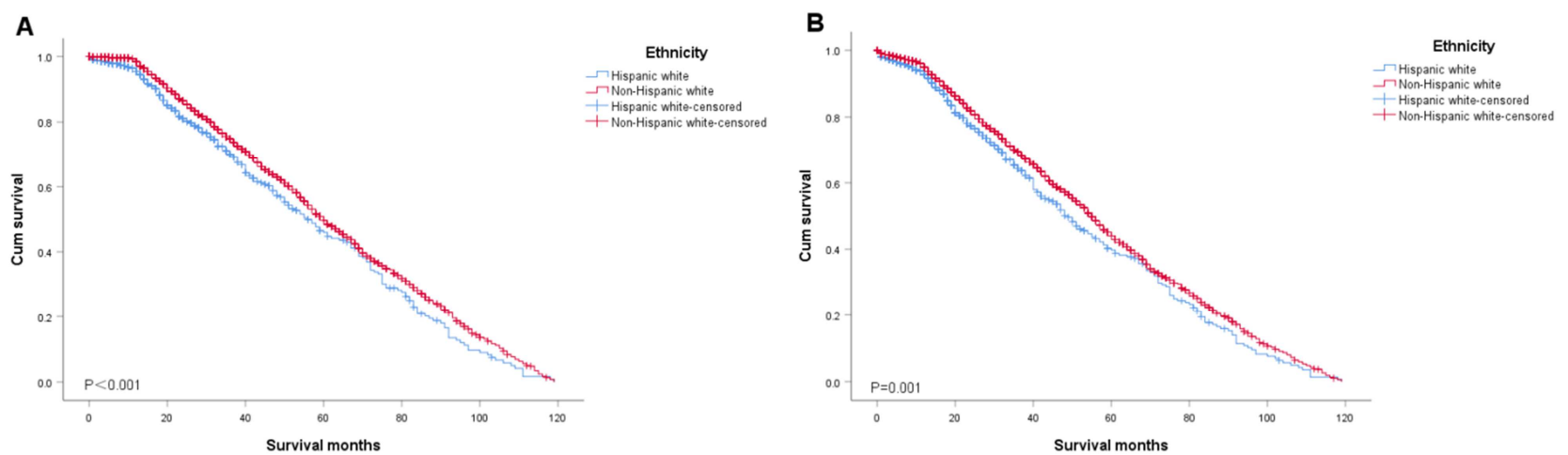

Figure 1. The survivorship curves of Hispanic and non-Hispanic whites with pancreatic head carcinoma were compared. (A) Overall survival. (B) Cause-specific survival. Cum, cumulative.

Table 1. Basic demographics and tumor features of sufferers from SEER database.

\begin{tabular}{|c|c|c|c|c|}
\hline Characteristic & Total & Hispanic White & Non-Hispanic White & P-value \\
\hline Patient, n(\%) & $9724(100.00)$ & $1351(13.89)$ & $8373(86.11)$ & \\
\hline Age, mean \pm SD & $66.49 \pm 11.22$ & $64.39 \pm 12.20$ & $66.82 \pm 11.02$ & $<0.001$ \\
\hline Year of diagnosis, n(\%) & & & & 0.003 \\
\hline $2007-2009$ & $3208(32.99)$ & $404(29.90)$ & $2095(25.02)$ & \\
\hline 2010-2012 & $3233(33.25)$ & $449(33.24)$ & $2804(33.49)$ & \\
\hline $2013-2015$ & $3283(33.76)$ & $498(36.86)$ & $3474(41.49)$ & \\
\hline Gender, $n(\%)$ & & & & 0.001 \\
\hline Female & $4579(47.09)$ & $660(48.85)$ & $3888(46.43)$ & \\
\hline Male & $5145(52.91)$ & $691(51.15)$ & $4485(53.57)$ & \\
\hline Marital status, n(\%) & & & & 0.012 \\
\hline Married & $6145(63.19)$ & $812(60.10)$ & $5333(63.69)$ & \\
\hline Unmarried & $3579(36.81)$ & $539(39.90)$ & $3040(36.31)$ & \\
\hline Grade, n(\%) & & & & 0.578 \\
\hline I & $1256(12.92)$ & $179(13.25)$ & $1077(12.86)$ & \\
\hline II & $4223(43.43)$ & $571(42.26)$ & $3652(43.62)$ & \\
\hline III & $4015(41.29)$ & $562(41.60)$ & $3453(41.24)$ & \\
\hline IV & $230(2.36)$ & $39(2.89)$ & $191(2.28)$ & \\
\hline AJCC TNM stage, $n(\%)$ & & & & $<0.001$ \\
\hline I & $788(8.10)$ & $113(8.36)$ & $675(8.06)$ & \\
\hline II & $5985(61.55)$ & $762(56.40)$ & $5223(62.38)$ & \\
\hline III & $789(8.12)$ & $111(8.22)$ & $678(8.10)$ & \\
\hline IV & $2162(22.23)$ & $365(27.02)$ & $1797(21.46)$ & \\
\hline Surgery, $n(\%)$ & & & & $<0.001$ \\
\hline Yes & $6049(62.21)$ & $759(56.18)$ & $5290(63.18)$ & \\
\hline No & $3675(37.79)$ & $592(43.82)$ & $3083(36.82)$ & \\
\hline Insurance status, $n(\%)$ & & & & $<0.001$ \\
\hline Insured & $8508(87.49)$ & $948(70.17)$ & $7560(90.29)$ & \\
\hline Medicaid & $986(10.14)$ & $343(25.39)$ & $643(7.68)$ & \\
\hline Uninsured & $230(2.37)$ & $60(4.44)$ & $170(2.03)$ & \\
\hline
\end{tabular}

Table 2. Univariate along with multivariate tabulated interpretation of OS amongst sufferers confirmed to pancreatic head carcinoma.

\begin{tabular}{|c|c|c|c|c|c|c|c|}
\hline \multirow[b]{2}{*}{ Characteristic } & \multicolumn{3}{|c|}{ Univariate analysis } & \multirow[b]{2}{*}{ P-value } & \multicolumn{3}{|c|}{ Multivariate analysis } \\
\hline & 5-year OS, \% & HR & $95 \% \mathrm{CI}$ & & HR & $95 \% \mathrm{CI}$ & P-value \\
\hline $\begin{array}{l}\text { Year of diagnosis } \\
2007-2009\end{array}$ & 97.6 & Reference & & & Reference & & \\
\hline $2010-2012$ & 58.0 & 30.745 & $21.409-44.152$ & $<0.001$ & 30.417 & $21.169-43.705$ & $<0.001$ \\
\hline $2013-2015$ & N/A & 1254.822 & $808.107-1948.477$ & $<0.001$ & 1257.477 & $809.476-1953.424$ & $<0.001$ \\
\hline $\begin{array}{l}\text { Age of diagnosis, } \\
\leq 59\end{array}$ & 49.8 & Reference & & & Reference & & \\
\hline $60-74$ & 48.0 & 1.087 & $0.978-1.209$ & 0.12 & 1.012 & $0.909-1.126$ & 0.833 \\
\hline$>74$ & 51.4 & 0.996 & $0.864-1.149$ & 0.961 & 1.053 & $0.91-1.217$ & 0.489 \\
\hline $\begin{array}{l}\text { Gender } \\
\text { Female }\end{array}$ & 48.9 & Reference & & & Reference & & \\
\hline $\begin{array}{l}\text { Male } \\
\text { Grade }\end{array}$ & 49.3 & 0.942 & $0.857-1.036$ & 0.218 & 1.025 & $0.93-1.13$ & 0.618 \\
\hline
\end{tabular}




\begin{tabular}{|c|c|c|c|c|c|c|c|}
\hline \multirow[b]{2}{*}{ Characteristic } & \multicolumn{3}{|c|}{ Univariate analysis } & \multirow[b]{2}{*}{ P-value } & \multicolumn{3}{|c|}{ Multivariate analysis } \\
\hline & 5-year OS,\% & HR & $95 \%$ CI & & HR & $95 \%$ CI & P-value \\
\hline I & 48.2 & Reference & & & Reference & & \\
\hline II & 49.5 & 0.994 & $0.883-1.119$ & 0.925 & 1.031 & $0.913-1.165$ & 0.622 \\
\hline III & 50.6 & 0.985 & $0.862-1.124$ & 0.818 & 1.014 & $0.885-1.163$ & 0.838 \\
\hline IV & 34.3 & 1.167 & $0.795-1.712$ & 0.43 & 0.742 & $0.503-1.094$ & 0.132 \\
\hline \multicolumn{8}{|l|}{ AJCC TNM } \\
\hline I & 49.0 & Reference & & & Reference & & \\
\hline II & 50.1 & 1.133 & $0.995-1.291$ & 0.06 & 1.093 & $0.955-1.252$ & 0.195 \\
\hline III & 45.3 & 1.056 & $0.804-1.388$ & 0.694 & 0.994 & $0.751-1.317$ & 0.969 \\
\hline IV & 45.0 & 1.392 & $1.13-1.715$ & 0.002 & 1.112 & $0.881-1.404$ & 0.372 \\
\hline \multicolumn{8}{|l|}{ Surgery } \\
\hline Yes & 49.6 & Reference & & & Reference & & \\
\hline No & 44.7 & 1.151 & $0.989-1.339$ & 0.068 & 1.143 & $0.955-1.367$ & 0.145 \\
\hline \multicolumn{8}{|l|}{ Race } \\
\hline Hispanic White & 45.9 & Reference & & & Reference & & \\
\hline $\begin{array}{l}\text { Non-Hispanic White } \\
\text { Marital status }\end{array}$ & 49.6 & 0.781 & $0.686-0.89$ & $<0.001$ & 0.832 & $0.728-0.951$ & 0.007 \\
\hline Married & 49.0 & Reference & & & Reference & & \\
\hline Unmarried & 49.4 & 0.962 & $0.869-1.064$ & 0.45 & 0.975 & $0.877-1.083$ & 0.634 \\
\hline \multicolumn{8}{|l|}{ Insurance status } \\
\hline Insured & 49.6 & Reference & & & Reference & & \\
\hline Medicaid & 46.0 & 1.157 & $0.969-1.381$ & 0.106 & 1.201 & $1-1.442$ & 0.051 \\
\hline Uninsured & 43.1 & 1.46 & $1.104-1.93$ & 0.008 & 1.482 & $1.115-1.968$ & 0.007 \\
\hline
\end{tabular}

OS, overall survival; HR, hazard ratio; CI, confidence interval; AJCC, American Joint Committee on Cancer; N/A, not available; TNM, Tumor-Node-Metastasis.

Table 3. Univariate along with multivariate tabulated interpretation of OS amongst sufferers confirmed to pancreatic head carcinoma.

\begin{tabular}{|c|c|c|c|c|c|c|c|}
\hline & & & Univariate analysis & & & Multivariate analysis & \\
\hline Characteristic & 5-year CSS, \% & HR & $95 \% \mathrm{CI}$ & P-value & HR & $95 \% \mathrm{CI}$ & P-value \\
\hline \multicolumn{8}{|l|}{ Year of diagnosis } \\
\hline $2007-2009$ & 83.9 & Reference & & & Reference & & \\
\hline $2010-2012$ & 52.5 & 3.954 & $3.352-4.663$ & $<0.001$ & 3.926 & $3.326-4.634$ & $<0.001$ \\
\hline 2013-2015 & N/A & 29.288 & $24.28-35.33$ & $<0.001$ & 29.412 & $24.369-35.498$ & $<0.001$ \\
\hline \multicolumn{8}{|l|}{ Age of diagnosis, years } \\
\hline$\leq 59$ & 46.5 & Reference & & & Reference & & \\
\hline $60-74$ & 42.6 & 1.167 & $1.059-1.287$ & 0.002 & 1.132 & $1.025-1.25$ & 0.014 \\
\hline$>74$ & 40.7 & 1.3 & $1.151-1.467$ & $<0.001$ & 1.357 & $1.198-1.536$ & $<0.001$ \\
\hline \multicolumn{8}{|l|}{ Gender } \\
\hline Female & 43.9 & Reference & & & Reference & & \\
\hline Male & 43.0 & 1.077 & $0.99-1.172$ & 0.085 & 1.078 & $0.988-1.176$ & 0.092 \\
\hline \multicolumn{8}{|l|}{ Grade } \\
\hline I & 42.9 & Reference & & & Reference & & \\
\hline II & 44.0 & 0.994 & $0.892-1.108$ & 0.915 & 1.009 & $0.903-1.128$ & 0.869 \\
\hline III & 44.1 & 1.031 & $0.916-1.161$ & 0.613 & 1.038 & $0.919-1.173$ & 0.547 \\
\hline IV & 29.6 & 1.091 & $0.77-1.545$ & 0.625 & 0.828 & $0.583-1.176$ & 0.291 \\
\hline \multicolumn{8}{|l|}{ AJCC TNM } \\
\hline I & 42.8 & Reference & & & Reference & & \\
\hline II & 44.5 & 1.075 & $0.956-1.21$ & 0.228 & 1.063 & $0.941-1.201$ & 0.323 \\
\hline III & 40.3 & 1.129 & $0.901-1.415$ & 0.292 & 1.061 & $0.84-1.34$ & 0.622 \\
\hline IV & 39.5 & 1.269 & $1.058-1.522$ & 0.01 & 1.034 & $0.844-1.267$ & 0.747 \\
\hline \multicolumn{8}{|l|}{ Surgery } \\
\hline Yes & 44.2 & Reference & & & Reference & & \\
\hline No & 38.8 & 1.273 & $1.126-1.439$ & $<0.001$ & 1.263 & $1.09-1.463$ & 0.002 \\
\hline \multicolumn{8}{|l|}{ Race } \\
\hline Hispanic White & 39.8 & Reference & & & Reference & & \\
\hline \multicolumn{7}{|l|}{ Marital status } & 0.037 \\
\hline Married & 44.0 & Reference & & & Reference & & \\
\hline Unmarried & 42.5 & 1.067 & $0.976-1.166$ & 0.153 & 1.067 & $0.972-1.171$ & 0.171 \\
\hline \multicolumn{8}{|l|}{ Insurance status } \\
\hline Insured & 44.2 & Reference & & & Reference & & \\
\hline Medicaid & 36.4 & 1.348 & $1.165-1.559$ & $<0.001$ & 1.371 & $1.178-1.596$ & $<0.001$ \\
\hline Uninsured & 39.1 & 1.383 & $1.072-1.785$ & 0.013 & 1.489 & $1.15-1.928$ & 0.003 \\
\hline
\end{tabular}

CSS, cause-specific survival; HR, hazard ratio; CI, confidence interval; AJCC, American Joint Committee on Cancer; N/A, not available; TNM, Tumor-Node-Metastasis. 


\subsection{Discrepancies in Surgery Status by Race}

The relevance of race to survival was also assessed based on whether or not the patients had received surgery. Figure 2 shows Kaplan-Meier survival curves for race and surgery. The 5 -year OS rate among patients who received surgery was superior for NHWs than HWs (49.9\% versus $47.5 \%, P=0.017$ ), as was the 5 -year CSS rate $(44.5 \%$ versus $41.7 \%, P=0.042)$. The 5-year OS rate remained superior for NHWs (46.6\%) over
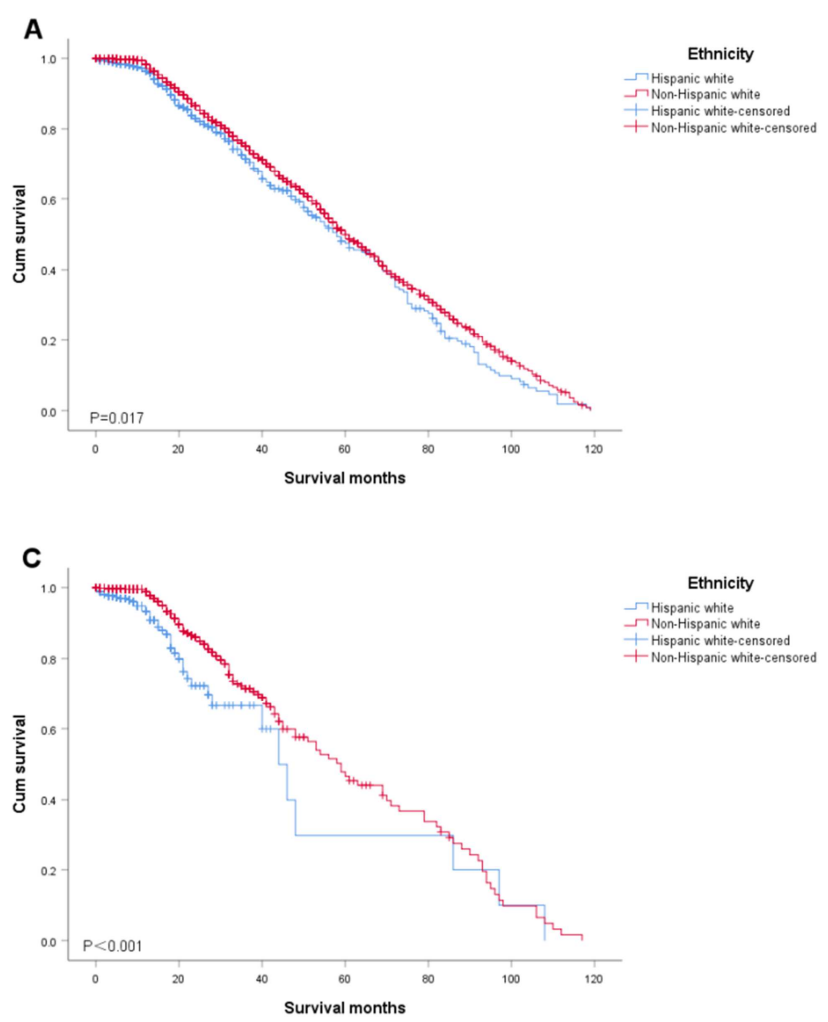

HWs $(30.0 \%, P<0.001)$ who had not received surgery, as did the CSS rate $(41.1 \%$ versus $22.9 \%, P=0.002)$. Multivariate analysis indicated that OS was affected by race both among those who received surgery $(\mathrm{HR}=0.843,95 \% \mathrm{CI}=0.731-0.972$, $P=0.018)$ and did not receive surgery $(\mathrm{HR}=0.491,95 \%$ $\mathrm{CI}=0.351-0.686, P<0.001)$, as was CSS $(\mathrm{HR}=0.874,95 \%$ $\mathrm{CI}=0.767-0.997, P=0.045$; and $\mathrm{HR}=0.653,95 \% \mathrm{CI}=0.495$ $0.861, P=0.003$; respectively) (Table 4 ).
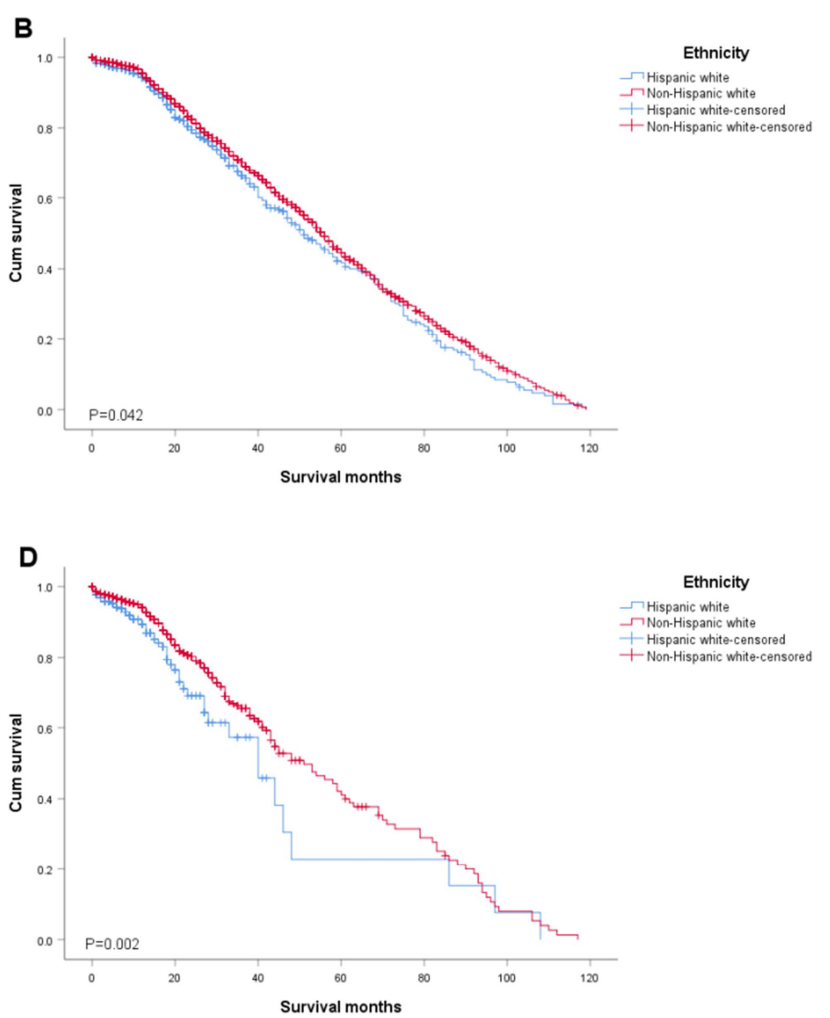

Figure 2. Survivorship curves of pancreatic head carcinoma sufferers of different races in two different surgical branches. (A) Surgical patient OS. (B) Surgical patient CSS (C) No surgical patient OS. (D) No surgical patient CSS. OS, overall survival; CSS, cause-specific survival; Cum, cumulative.

Table 4. Tabulated interpretation of univariate and multifactorial survivorship for pancreatic head carcinoma sufferers drawn on surgery.

\begin{tabular}{|c|c|c|c|c|c|c|c|}
\hline \multicolumn{8}{|l|}{$\mathrm{A}, \mathrm{OS}$} \\
\hline \multirow[b]{2}{*}{ Characteristic } & \multirow[b]{2}{*}{ 5-year survival, $\%$} & \multirow[b]{2}{*}{ Median survival time, months } & \multicolumn{2}{|l|}{ Univariate analysis } & \multicolumn{3}{|c|}{ Multivariate analysis } \\
\hline & & & Log $\operatorname{rank} \chi 2$ & P-value & HR & $95 \% \mathrm{CI}$ & P-value \\
\hline Surgery (Yes) & & & 5.721 & 0.017 & & & \\
\hline Hispanic White & 47.5 & 58 & & & \multicolumn{2}{|l|}{ Reference } & \\
\hline Non-Hispanic White & 49.9 & 60 & & & 0.843 & $0.731-0.972$ & 0.018 \\
\hline Surgery (No) & & & 18.342 & $<0.001$ & & & \\
\hline \multirow{3}{*}{$\begin{array}{l}\text { Non-Hispanic White } \\
\text { B, CSS }\end{array}$} & 46.6 & 49 & & & 0.491 & $0.351-0.686$ & $<0.001$ \\
\hline & & & & & & & \\
\hline & & & Univariate analysis & & \multicolumn{2}{|c|}{ Multivariate analysis } & \\
\hline Characteristic & \multirow[t]{2}{*}{ 5-year survival, $\%$} & \multirow[t]{2}{*}{ Median survival time, months } & Log rank $\chi 2$ & P-value & \multirow[t]{2}{*}{ HR } & \multirow[t]{2}{*}{$95 \% \mathrm{CI}$} & \multirow[t]{2}{*}{ P-value } \\
\hline Surgery (Yes) & & & 4.136 & 0.042 & & & \\
\hline Hispanic White & 41.7 & 51 & & & \multicolumn{2}{|l|}{ Reference } & \\
\hline \multicolumn{2}{|l|}{ Surgery (No) } & & 9.373 & 0.002 & & & \\
\hline Hispanic White & 22.9 & 40 & & & \multicolumn{2}{|l|}{ Reference } & \\
\hline Non-Hispanic White & 41.1 & 51 & & & 0.653 & $0.495-0.861$ & 0.003 \\
\hline
\end{tabular}




\subsection{Discrepancies in Insurance Status by Race}

The Kaplan-Meier survival curves for the effects of insurance status are shown in Figure 3. The 5-year OS rate did not differ significantly between insured HWs and insured NHW (51.9\% versus $49.3 \%, P=0.293)$, and likewise for the 5 -year CSS rate $(45.9 \%$ versus $44.0 \%, P=0.792)$. However, the 5-year OS rate among Medicaid recipients was better in the NHW group (50.3\%) than the HW group (50.3\% versus $38.6 \%, P=0.001$ ), as was the 5 -year CSS rate (40.3\% versus $29.9 \%, P=0.005)$. Consistently, the 5 -year
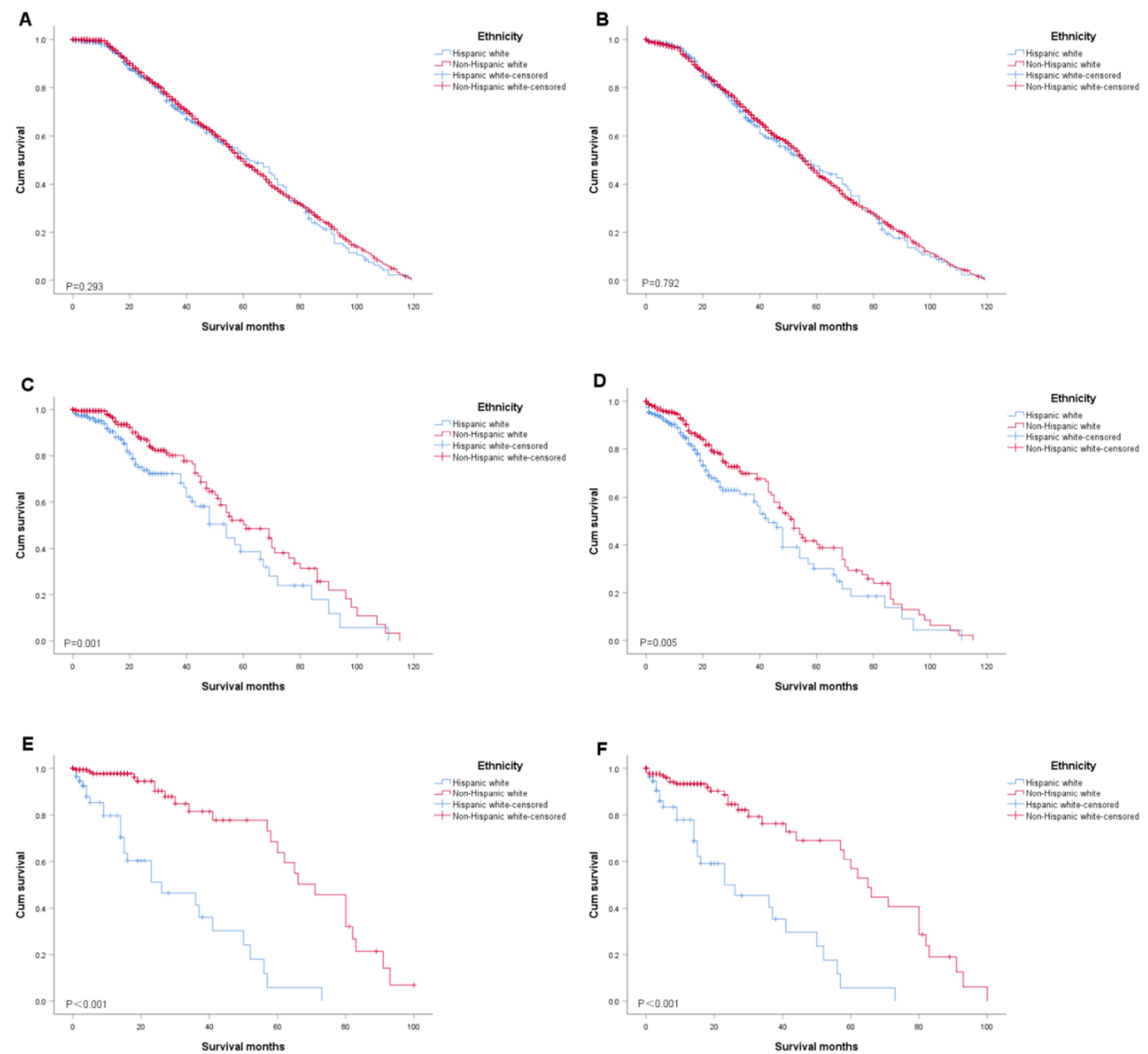

OS rate among uninsured NHWs was far better than that among HWs $(64.0 \%$ versus $6.0 \%, P<0.001)$, as was the 5-year CSS rate $(57.0 \%$ versus $5.9 \%, \quad P<0.001)$. Multivariate analysis showed that OS was influenced by race not only among Medicaid recipients (HR $=0.574,95 \%$ $\mathrm{CI}=0.407-0.81, \quad P=0.002) \quad$ but also among uninsured patients $(\mathrm{HR}=0.289,95 \% \mathrm{CI}=0.083-0.289, P<0.001)$, and similarly for CSS $(\mathrm{HR}=0.669, \quad 95 \% \quad \mathrm{CI}=0.505-0.887$, $P=0.005$; and $\mathrm{HR}=0.233,95 \% \mathrm{CI}=0.134-0.404, P<0.001$; respectively) (Table 5).

Figure 3. Survivorship curves in three Insurance status branchings sufferers with pancreatic head carcinoma in accordance with ethnicity. (A) Insured OS. (B) Insured CSS. (C) Any Medicaid OS. (D) Any Medicaid CSS. (E) Uninsured OS. (F) Uninsured CSS. OS, overall survival; CSS, cause-specific survival; Cum, cumulative. 
Hongyu Yu et al:: Survival Advantage of Non-hispanic White Patients with Pancreatic Head Carcinoma: A Population-based Study

Table 5. Tabulated interpretation of univariate and multifactorial survival for pancreatic head carcinoma sufferers drawn on insurance status.

\begin{tabular}{|c|c|c|c|c|c|c|c|}
\hline \multicolumn{8}{|l|}{ A, OS } \\
\hline \multirow[b]{2}{*}{ Characteristic } & \multirow[b]{2}{*}{ 5-year survival, \% } & \multirow[b]{2}{*}{ Median survival time, months } & \multicolumn{2}{|c|}{ Univariate analysis } & \multicolumn{3}{|c|}{ Multivariate analysis } \\
\hline & & & Log rank $\chi_{2}$ & P-value & HR & $95 \% \mathrm{CI}$ & P-value \\
\hline Insured & & & 1.106 & 0.293 & & & \\
\hline Hispanic White & 51.9 & 62 & & & Reference & & \\
\hline Non-Hispanic White & 49.3 & 60 & & & 0.921 & $0.789-1.076$ & 0.3 \\
\hline Medicaid & & & 10.415 & 0.001 & & & \\
\hline Hispanic White & 38.6 & 54 & & & Reference & & \\
\hline Non-Hispanic White & 50.3 & 61 & & & 0.574 & $0.407-0.81$ & 0.002 \\
\hline Uninsured & & & 44.871 & $<0.001$ & & & \\
\hline Hispanic White & 6.0 & 26 & & & Reference & & \\
\hline $\begin{array}{l}\text { Non-Hispanic White } \\
\text { B, CSS }\end{array}$ & 64.0 & 71 & & & 0.289 & $0.083-0.289$ & $<0.001$ \\
\hline & & & \multicolumn{2}{|c|}{ Univariate analysis } & \multicolumn{2}{|c|}{ Multivariate analysis } & \\
\hline $\begin{array}{l}\text { Characteristic } \\
\text { Insured }\end{array}$ & 5-year survival, $\%$ & Median survival time, months & $\begin{array}{l}\text { Log rank } \chi 2 \\
0.07\end{array}$ & $\begin{array}{l}\text { P-value } \\
0.792\end{array}$ & $\mathrm{HR}$ & $95 \% \mathrm{CI}$ & P-value \\
\hline Hispanic White & 45.9 & 55 & & & Reference & & \\
\hline Non-Hispanic White & 44.0 & 55 & & & 0.981 & $0.851-1.132$ & 0.794 \\
\hline Medicaid & & & 8.064 & 0.005 & & & \\
\hline Hispanic White & 29.9 & 43 & & & Reference & & \\
\hline Non-Hispanic White & 40.3 & 52 & & & 0.669 & $0.505-0.887$ & 0.005 \\
\hline Uninsured & & & 31.729 & $<0.001$ & & & \\
\hline Hispanic White & 5.9 & 26 & & & Reference & & \\
\hline Non-Hispanic White & 57.0 & 65 & & & 0.233 & $0.134-0.404$ & $<0.001$ \\
\hline OS, overall survival; & S, cause-specific sur & $1 ; \mathrm{HR}$, hazard ratio; CI, confifi & erval. & & & & \\
\hline
\end{tabular}

\subsection{Discrepancies in AJCC TNM Stage by Race}

Figure 4 shows Kaplan-Meier survival curves for the relationships between race and AJCC TNM stages I, II, III, and IV ( $\mathrm{n}=788,5985,789$, and 2162 respectively). Among those at AJCC TNM stage II, both the 5-year OS $(P=0.003)$ and CSS $(P=0.001)$ rates were higher in the NHW group $(50.5 \%$ and $45.2 \%$ respectively) than the HW group ( 46.8 and $39.1 \%$ respectively), whereas the survival rates did not differ significantly with race for AJCC TNM stage I, III, or IV. Multivariate analysis showed that race was an independent prognostic factor for $\mathrm{OS}(\mathrm{HR}=0.787,95 \%$ $\mathrm{CI}=0.671-0.925, \quad P=0.004)$ and $\mathrm{CSS} \quad(\mathrm{HR}=0.789, \quad 95 \%$ $\mathrm{CI}=0.683-0.911, P=0.001)$ among patients at AJCC TNM stage II, but not among those at AJCC TNM stage I, III, or IV (Table 6).

Table 6. Tabulated interpretation of univariate and multifactorial survivorship for pancreatic head carcinoma sufferers drawn on the AJCC TNM stage.

\begin{tabular}{|c|c|c|c|c|c|c|c|}
\hline \multicolumn{8}{|l|}{$\mathbf{A}, \mathrm{OS}$} \\
\hline \multirow[b]{2}{*}{ Characteristic } & \multirow[b]{2}{*}{ 5-year survival, \% } & \multirow[b]{2}{*}{ Median survival time, months } & \multicolumn{2}{|c|}{ Univariate analysis } & \multicolumn{3}{|c|}{ Multivariate analysis } \\
\hline & & & Log $\operatorname{rank} \chi^{2}$ & P-value & HR & $95 \% \mathrm{CI}$ & P-value \\
\hline Stage I & & & 1.143 & 0.285 & & & \\
\hline Hispanic White & 47.6 & 58 & & & Reference & & \\
\hline Non-Hispanic White & 49.3 & 60 & & & 0.852 & $0.632-1.149$ & 0.293 \\
\hline Stage II & & & 8.774 & 0.003 & & & \\
\hline Hispanic White & 46.8 & 54 & & & Reference & & \\
\hline Non-Hispanic White & 50.5 & 61 & & & 0.787 & $0.671-0.925$ & 0.004 \\
\hline Stage III & & & 3.711 & 0.054 & & & \\
\hline Hispanic White & 49.8 & 45 & & & Reference & & \\
\hline Non-Hispanic White & 45.4 & 59 & & & 0.519 & $0.262-1.027$ & 0.06 \\
\hline Stage IV & & & 3.084 & 0.079 & & & \\
\hline Hispanic White & 43.6 & 60 & & & Reference & & \\
\hline $\begin{array}{l}\text { Non-Hispanic White } \\
\mathrm{B}, \mathrm{CSS}\end{array}$ & 45.4 & 55 & & & 0.697 & $0.463-1.05$ & 0.084 \\
\hline & & & Univariate an & alysis & Multivariat & analysis & \\
\hline $\begin{array}{l}\text { Characteristic } \\
\text { Stage I }\end{array}$ & 5-year survival, $\%$ & Median survival time, months & $\begin{array}{l}\text { Log rank } \chi^{2} \\
0.07\end{array}$ & $\begin{array}{l}\text { P-value } \\
0.791\end{array}$ & HR & $95 \% \mathrm{CI}$ & P-value \\
\hline Hispanic White & 44.6 & 57 & & & Reference & & \\
\hline Non-Hispanic White & 42.4 & 55 & & & 0.963 & $0.726-1.277$ & 0.794 \\
\hline Stage II & & & 10.775 & 0.001 & & & \\
\hline Hispanic White & 39.1 & 47 & & & Reference & & \\
\hline Non-Hispanic White & 45.2 & 56 & & & 0.789 & $0.683-0.911$ & 0.001 \\
\hline Stage III & & & 1.139 & 0.286 & & & \\
\hline Hispanic White & 48.2 & 45 & & & Reference & & \\
\hline Non-Hispanic White & 40.0 & 52 & & & 0.728 & $0.404-1.311$ & 0.29 \\
\hline
\end{tabular}




\begin{tabular}{|c|c|c|c|c|c|c|c|}
\hline \multicolumn{8}{|l|}{$\mathrm{A}, \mathrm{OS}$} \\
\hline \multirow[b]{2}{*}{ Characteristic } & \multirow[b]{2}{*}{ 5-year survival, $\%$} & \multirow[b]{2}{*}{ Median survival time, months } & \multicolumn{2}{|c|}{ Univariate analysis } & \multicolumn{3}{|c|}{ Multivariate analysis } \\
\hline & & & Log rank $\chi^{2}$ & P-value & HR & $95 \%$ CI & P-value \\
\hline Stage IV & & & 0.519 & 0.471 & & & \\
\hline Hispanic White & 37.3 & 48 & & & Reference & & \\
\hline Non-Hispanic White & 40.0 & 49 & & & 0.874 & $0.604-1.265$ & 0.476 \\
\hline
\end{tabular}
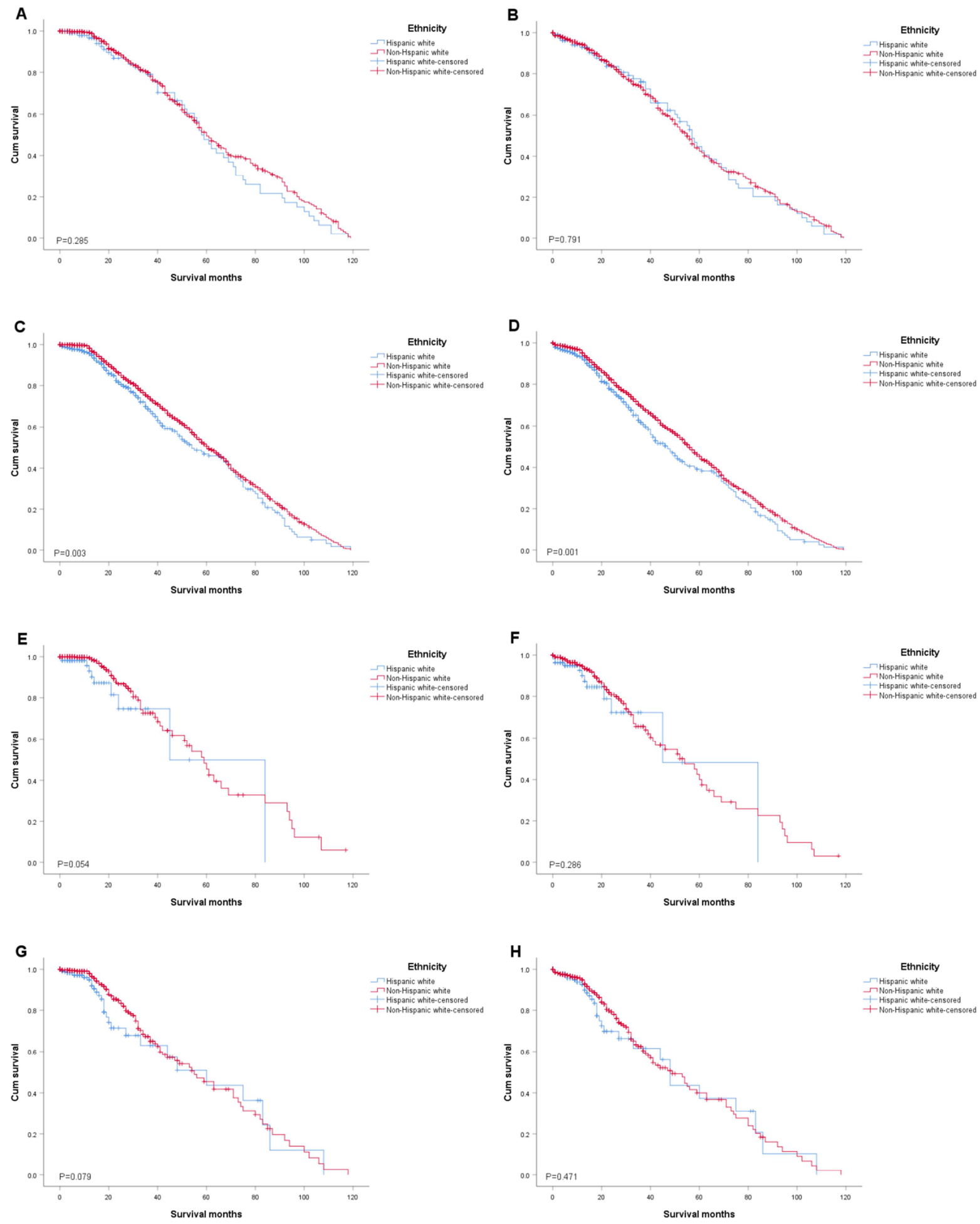

Figure 4. Survivorship curves of pancreatic head carcinoma sufferers at distinguished Tumor-Node-Metastasis stages in accordance with ethnicity. (A) Stage-I sufferers' OS. (B) Stage-I sufferers' CSS. (C) Stage-II sufferers' OS. (D) Stage-II sufferers' CSS. (E) Stage-III sufferers' OS. (F) Stage-III sufferers' CSS. (G) Stag-IV sufferers' OS. (H) Stage-IV sufferers' CSS. OS, overall survival; CSS, cause-specific survival; Cum, cumulative. 


\section{Discussion}

$\mathrm{PC}$ is an aggressive disease whose mortality rate has been increasingly improved [14]. Interventions implemented over the past 2 decades have failed to improve the 5-year OS for $\mathrm{PC}$, which is known to be the deadliest solid tumor [15]. Moreover, PHC represents the predominant form of $\mathrm{PC}$ and has the worst prognosis [16]. With the aim of clarifying the correlation between race and survival outcomes of PHC patients, the present study obtained information from the SEER database. Both OS and CSS rates were better among NHW than HW patients in univariate and multivariate analyses. Race was found to be an independent prognostic factor for survival among PHC patients, revealing that the epidemiological paradoxes are not entirely credible, which contrasts the results obtained by Ashktorab et al. [17].

In general, more PHC patients had received surgery, which is an important intervention in this population [18]. In the current study, a larger proportion of NHW than HW patients had received surgery. Multiple factors affect whether patients receive surgery, with the decision of a patient to reject surgical treatment remaining unclear [19]. The present analyses of the interactions between race and the surgery status in PHC patients found that both OS (surgery received: $\mathrm{HR}=0.843,95 \% \mathrm{CI}=0.731-0.972, \quad P=0.018$; surgery not received: $\mathrm{HR}=0.491,95 \% \mathrm{CI}=0.351-0.686, P<0.001)$ and CSS (surgery received: HR $=0.874,95 \% \mathrm{CI}=0.767-0.997$, $P=0.045$; surgery not received: $\mathrm{HR}=0.653,95 \% \mathrm{CI}=0.495$ $0.861, P=0.003$ ) were better in NHW patients than HW patients. These results are similar to the finding of Nipp et al. [20].

Other studies have found discrepancies between cancer diagnosis and treatment, and that the OS can be improved if access to health care is improved [21]. Our study found that both the 5-year OS and CSS were better for insured patients than for those with Medicaid and without insurance. Abraham et al. found that the insurance status of PC patients did not vary among blacks, whites, and other races [22], whereas when we divided the patients into HWs and NHWs, the latter showed a survival advantage regardless of whether they had Medicaid or were uninsured.

Non-Hispanics are reportedly more likely to have stage IIB disease than Hispanics [23]. Also, our subgroup analysis of AJCC TNM stage revealed that for both the OS $(\mathrm{HR}=0.787$, 95\% CI $=0.671-0.925, P=0.004)$ and $\mathrm{CSS}(\mathrm{HR}=0.789,95 \%$ $\mathrm{CI}=0.683-0.911, P=0.001)$, NHW race is an independent prognostic factor among patients at AJCC TNM stage II, but not among those at AJCC TNM stage I, III, or IV. Therefore, race may be a notable protective factor for patients in the early stage.

The survival advantage in NHW patients mentioned above provides reference information for the prophylaxis and cure of PHC. Strategies to reduce the likelihood of cancer in the Hispanic population include targeted, culturally appropriate interventions that increase access to preventive services and reduce the prevalence of cancer risk factors, as well as extra financial support [24]. Since most patients die from distant metastasis, it is not sufficient to restrict treatment to the primary site when attempting to improve the survival rate of these patients; instead, effective treatment that addresses systemic metastasis is crucial [25]. It is necessary to determine how to encourage patients who need surgery to participate in making informed, shared decisions [26]. Consideration of the cultural and psychological environments is particularly important. Our study has indicated that interventions should be somewhat more focused on improving treatment compliance in HW patients. Prevention, early diagnosis, improved treatment, and drugs also form important parts of the broad strategy to reduce the incidence of this cancer and improve its prognosis [27].

A distinct increased risk of innate PC can be seen in close relatives of patients confirmed as having inherited PC, hereditary cancer syndrome, or genetic pancreatitis. Knowledge of risk, benefit, and outcome data allow patients to make personalized decisions about screening and monitoring strategies [27], and the interconnectedness of racial groups should also not be overlooked. Moreover, economic factors such as the type of insurance and the income level are related to whether patients receive adjuvant chemotherapy. Therefore, when a patient receives adjuvant chemotherapy, the decision to use multiple drugs or a single drug is more likely to be influenced by patient or provider bias than by cost issues. Moreover, receiving multidrug chemotherapy seems to be associated with demographic variables such as race [28]. This means that racial disparities can be narrowed by targeting certain populations, expanding insurance coverage, and increasing Medicaid subsidies.

The limitations of this study were as follows: (i) there have been very few studies specifically on PHC, and so most of the cited literature was related to PC in the hope that this would include data on PHC; (ii) the small amount of information on comorbidities and relapses in the SEER database makes it difficult to compare the findings of effectiveness analyses [29]; and (iii) if the data registrars had not been aware of changes in the socioeconomic status of cases or even missed or incorrectly recorded this information, the study might have been influenced by the sources of these errors and biases [30].

\section{Conclusion}

In summary, race appears to be an independent prognostic factor for the OS and CSS of PHC patients. The survival rate of NHW patients was superior to that of HW patients, with this racial advantage evident in patients in the early stage of disease, who had received surgery, and who had insurance or Medicaid.

\section{Acknowledgements}

We would like to express our sincere gratitude to Professor 
Lv Jun and his big data research team on account of helping us to cultivate data thinking and master data mining methods.

\section{References}

[1] Wang W, He Y, Wu L, et al. Efficacy of extended versus standard lymphadenectomy in pancreatoduodenectomy for pancreatic head adenocarcinoma. An update meta-analysis. Pancreatology. 2019 Dec; 19 (8): 1074-1080. doi: 10.1016/j.pan.2019.10.003. PubMed PMID: 31668841.

[2] Moutinho-Ribeiro P, Coelho R, Giovannini M, et al. Pancreatic cancer screening: Still a delusion? Pancreatology. 2017 Sep Oct; 17 (5): 754-765. doi: 10.1016/j.pan.2017.07.001. PubMed PMID: 28739291.

[3] Cheon YK, Koo JK, Lee YS, et al. Elevated hemoglobin A1c levels are associated with worse survival in advanced pancreatic cancer patients with diabetes. Gut Liver. 2014 Mar; 8 (2): 205-14. doi: 10.5009/gnl.2014.8.2.205. PubMed PMID: 24672663; PubMed Central PMCID: PMCPMC3964272.

[4] Lesina M, Kurkowski MU, Ludes K, et al. Stat3/Socs3 activation by IL-6 transsignaling promotes progression of pancreatic intraepithelial neoplasia and development of pancreatic cancer. Cancer Cell. 2011 Apr 12; 19 (4): 456-69. doi: 10.1016/j.ccr.2011.03.009. PubMed PMID: 21481788.

[5] Ryan DP, Hong TS, Bardeesy N. Pancreatic adenocarcinoma. N Engl J Med. 2014 Sep 11; 371 (11): 1039-49. doi: 10.1056/NEJMra1404198. PubMed PMID: 25207767.

[6] Flores I, Casaletto KB, Marquine MJ, et al. Performance of Hispanics and Non-Hispanic Whites on the NIH Toolbox Cognition Battery: the roles of ethnicity and language backgrounds. Clin Neuropsychol. 2017 May; 31 (4): 783-797. doi: 10.1080/13854046.2016.1276216. PubMed PMID: 28080261; PubMed Central PMCID: PMCPMC5497573.

[7] Goldman N. Will the Latino Mortality Advantage Endure? Res Aging. 2016 Apr; 38 (3): 263-82. doi: 10.1177/0164027515620242. PubMed PMID: 26966251; PubMed Central PMCID: PMCPMC4955825.

[8] Arias E, Kochanek KD, Anderson RN. How Does Cause of Death Contribute to the Hispanic Mortality Advantage in the United States? NCHS Data Brief. 2015 Nov (221): 1-8. PubMed PMID: 26633554.

[9] Cervantes A, Waymouth EK, Petrov MS. African-Americans and Indigenous Peoples Have Increased Burden of Diseases of the Exocrine Pancreas: A Systematic Review and Meta-Analysis. Dig Dis Sci. 2019 Jan; 64 (1): 249-261. doi: 10.1007/s10620-018-5291-1. PubMed PMID: 30259278.

[10] Gagliardi AR, Soong D, Gallinger S. Identifying Factors Influencing Pancreatic Cancer Management to Inform Quality Improvement Efforts and Future Research: A Scoping Systematic Review. Pancreas. 2016 Feb; 45 (2): 161-6. doi: 10.1097/MPA.0000000000000484. PubMed PMID: 26752254.

[11] Yang J, Li Y, Liu Q, et al. Brief introduction of medical database and data mining technology in big data era. J Evid Based Med. 2020 Feb; 13 (1): 57-69. doi: 10.1111/jebm.12373. PubMed PMID: 32086994; PubMed Central PMCID: PMCPMC7065247.

[12] Torgeson A, Tao R, Garrido-Laguna I, et al. Large database utilization in health outcomes research in pancreatic cancer: an update. J Gastrointest Oncol. 2018 Dec; 9 (6): 996-1004. doi: 10.21037/jgo.2018.05.15. PubMed PMID: 30603118; PubMed Central PMCID: PMCPMC6286942.

[13] Mukhija D, Nagpal SJS, Sohal DPS. Epidemiology, Tumor Characteristics, and Survival in Patients With Primary Pancreatic Lymphoma: A Large Population-based Study Using the SEER Database. Am J Clin Oncol. 2019 May; 42 (5): 454-458. doi: 10.1097/COC.0000000000000544. PubMed PMID: 30950860.

[14] Henley SJ, Ward EM, Scott S, et al. Annual report to the nation on the status of cancer, part I: National cancer statistics. Cancer. 2020 May 15; 126 (10): 2225-2249. doi: 10.1002/cncr.32802. PubMed PMID: 32162336; PubMed Central PMCID: PMCPMC7299151.

[15] Yang R, Cheung MC, Byrne MM, et al. Survival effects of adjuvant chemoradiotherapy after resection for pancreatic carcinoma. Arch Surg. 2010 Jan; 145 (1): 49-56. doi: 10.1001/archsurg.2009.244. PubMed PMID: 20083754.

[16] El Nakeeb A, Roshdy S, Ask W, et al. Comparative Study between Uncinate process carcinoma and Pancreatic head carcinoma after Pancreaticodudenectomy (Clincopathological features and Surgical outcomes). Hepato-Gastroenterol. 2014 Sep; 61 (134): 1748-1755. PubMed PMID: WOS:000341170900046; English.

[17] Ashktorab H, Kupfer SS, Brim H, et al. Racial Disparity in Gastrointestinal Cancer Risk. Gastroenterology. 2017 Oct; 153 (4): 910-923. doi: 10.1053/j.gastro.2017.08.018. PubMed PMID: 28807841; PubMed Central PMCID: PMCPMC5623134.

[18] McGuigan A, Kelly P, Turkington RC, et al. Pancreatic cancer: A review of clinical diagnosis, epidemiology, treatment and outcomes. World J Gastroenterol. 2018 Nov 21; 24 (43): 4846-4861. doi: 10.3748/wjg.v24.i43.4846. PubMed PMID: 30487695; PubMed Central PMCID: PMCPMC6250924.

[19] Molina G, Clancy TE, Tsai TC, et al. Racial Disparity in Pancreatoduodenectomy for Borderline Resectable Pancreatic Adenocarcinoma. Ann Surg Oncol. 2020 Jul 10. doi: 10.1245/s10434-020-08717-x. PubMed PMID: 32651695.

[20] Nipp R, Tramontano AC, Kong CY, et al. Disparities in cancer outcomes across age, sex, and race/ethnicity among patients with pancreatic cancer. Cancer Med. 2018 Feb; 7 (2): 525-535. doi: 10.1002/cam4.1277. PubMed PMID: 29322643; PubMed Central PMCID: PMCPMC5806100.

[21] Loehrer AP, Chang DC, Hutter MM, et al. Health Insurance Expansion and Treatment of Pancreatic Cancer: Does Increased Access Lead to Improved Care? J Am Coll Surg. 2015 Dec; 221 (6): 1015-22. doi: 10.1016/j.jamcollsurg.2015.09.010. PubMed PMID: 26611798; PubMed Central PMCID: PMCPMC4662773.

[22] Abraham A, Al-Refaie WB, Parsons HM, et al. Disparities in pancreas cancer care. Ann Surg Oncol. 2013 Jun; 20 (6): 2078-87. doi: 10.1245/s10434-012-2843-z. PubMed PMID: 23579872 .

[23] Shapiro M, Chen Q, Huang Q, et al. Associations of Socioeconomic Variables With Resection, Stage, and Survival in Patients With Early-Stage Pancreatic Cancer. JAMA Surg. 2016 Apr; 151 (4): 338-45. doi: 10.1001/jamasurg.2015.4239. PubMed PMID: 26581025. 
[24] Miller KD, Goding Sauer A, Ortiz AP, et al. Cancer Statistics for Hispanics/Latinos, 2018. CA Cancer J Clin. 2018 Nov; 68 (6): 425-445. doi: 10.3322/caac.21494. PubMed PMID: 30285281 .

[25] Nordby T, Hugenschmidt H, Fagerland MW, et al. Follow-up after curative surgery for pancreatic ductal adenocarcinoma: asymptomatic recurrence is associated with improved survival. Eur J Surg Oncol. 2013 Jun; 39 (6): 559-66. doi: 10.1016/j.ejso.2013.02.020. PubMed PMID: 23498362.

[26] Ziebland S, Chapple A, Evans J. Barriers to shared decisions in the most serious of cancers: a qualitative study of patients with pancreatic cancer treated in the UK. Health Expect. 2015 Dec; 18 (6): 3302-12. doi: 10.1111/hex.12319. PubMed PMID: 25496598; PubMed Central PMCID: PMCPMC5810685.

[27] Sohal DPS, Willingham FF, Falconi M, et al. Pancreatic Adenocarcinoma: Improving Prevention and Survivorship. Am Soc Clin Oncol Educ Book. 2017; 37: 301-310. doi: 10.14694/EDBK_175222 10.1200/EDBK_175222. PubMed PMID: 28561672.
[28] Sanford NN, Aguilera TA, Folkert MR, et al. Sociodemographic Disparities in the Receipt of Adjuvant Chemotherapy Among Patients With Resected Stage I-III Pancreatic Adenocarcinoma. J Natl Compr Canc Netw. 2019 Nov 1; 17 (11): 1292-1300. doi: 10.6004/jnccn.2019.7322. PubMed PMID: 31693987.

[29] Doll KM, Rademaker A, Sosa JA. Practical Guide to Surgical Data Sets: Surveillance, Epidemiology, and End Results (SEER) Database. JAMA Surg. 2018 Jun 1; 153 (6): 588-589. doi: 10.1001/jamasurg.2018.0501. PubMed PMID: 29617544.

[30] Sun H, Ma H, Hong G, et al. Survival improvement in patients with pancreatic cancer by decade: a period analysis of the SEER database, 1981-2010. Sci Rep. 2014 Oct 23; 4: 6747. doi: 10.1038/srep06747. PubMed PMID: 25339498; PubMed Central PMCID: PMCPMC5381379. 